\title{
Biomineralogical Investigation of Apatite Piezoelectricity
}

\section{Pawlikowski M}

Laboratory of Biomineralogy, Department of Mineralogy, Petrography and Geochemistry, Faculty of Geology, Geophysics and Environmental Protection, AGH University of Science and Technology, al. Mickiewicza 30, 30-059 Krakow, Poland

*Corresponding author: Pawlikowski, Laboratory of Biomineralogy, Department of Mineralogy, Petrography and Geochemistry, Faculty of Geology, Geophysics and Environmental Protection, AGH University of Science and Technology, al. Mickiewicza 30, 30-059 Krakow, Poland, Tel: +48126172222; E-mail: mpawlik@uci.agh.edu.pl

Received date: June 16, 2016; Accepted date: June 17, 2016; Published date: June 20, 2016

Copyright: (c) 2016 Pawlikowski M. This is an open-access article distributed under the terms of the Creative Commons Attribution License, which permits unrestricted use, distribution, and reproduction in any medium, provided the original author and source are credited.

\begin{abstract}
Investigation of apatite piezoelectricity was conducted in order to assess piezoelectric properties of bone. In the first stage, mineralogical analysis of different apatite crystals, regarding their purity and fitness for the experiments was performed. After the crystals, had been chosen, $0.8 \mathrm{~mm}$-thick plates were cut, perpendicular and parallel to the crystallographic $Z$ axis. The plates were then polished and dusted with gold. Electrodes were attached to the opposite surfaces of the plates with conductive glue. So, prepared plates were hooked up to the EEG machine used for measuring electrical activity in the brain. The plates were then gently tapped to observe and register currents generated in them. Acquired data was processed by subtracting from the resulting graphs those generated by a hand movement, without tapping the plate. Results indicate that apatite plates have weak piezoelectric properties. Observed phenomenon may be translated to bone apatite, which would explain, at least partially, piezoelectric properties of bone. Acquired results suggest that there is a relation between the mechanical workload of bones (bone apatite) and their electrical properties. Considering the massive internal surface of bones, they may be treated as a kind of internal "antenna" reacting not only to mechanical stimuli, but to changes in electromagnetic field as well. Observed phenomena no doubt significantly influence the biological processes occurring in bones and the whole human body.
\end{abstract}

Keywords: Apatite; Piezoelectricity; Bone

\section{Introduction}

Considering the problem of piezoelectricity in bones, we need to remember that by weight, bones consist of 35\% type 1 collagen, and around $65 \%$ carbonated hydroxyapatite [1]. The phenomenon of piezoelectricity observed in bones has assuredly significant influence on their functioning, and indirectly, on functioning of the whole body. This phenomenon is exceedingly important due to the physicochemical equilibrium that occurs between the bone trabeculae and substances found in the spaces between the trabeculae. Presence of electrical phenomena in bones was unequivocally and doubtlessly proven [2-8]. That means that even minor mechanical impact on bones may change the electrical potentials inside and around them, which in turn affects the biochemical processes. Therefore, understanding the phenomenon of piezoelectricity in bones is an area of interest for many researchers. The phenomenon of piezoelectricity was discovered in the 1880s by Pierre and Jacques Curie. In the department of mineralogy of the Sorbonne University, they conducted a series of experiments which became a foundation for future publications [9-18]. Their early experiments, which were the effect of many theoretical discussions and mathematical analyses, were conducted on tourmaline crystals. Later they were continued on crystals of many other minerals, including quartz [19]. Their research proved that some crystals have the ability to change electrical energy into mechanical, and mechanical into electrical. The problem of the potentials occurring on long bones when they are being bent is a very interesting one [20-23]. Research conducted in this area by Delpech-Wolff and others $[2,24,25]$ suggest that bone collagen has piezoelectric properties. It is supposed to have been confirmed by experiments consisting of researching electrical phenomena in natural and demineralized (devoid of apatite) bones. They are supposed to show nearly identical "electrical" behavior in each case. Those experiments led dr. Wolff to assume that the piezoelectric phenomena in deformed bones are connected mostly to collagen. However, research by other scientists [1] suggests that collagen does not have piezoelectric properties after all. It was also suggested that the electrical potential in bones under pressure may be connected with the fluids inside the bone that are undergoing pressure [26-30]. Not much attention has been paid so far to the mineral bone hydroxyapatite. Apatite [29], similar to piezoelectric tourmalines and quartz, forms six-sided prism crystals. Like quartz, it is optically uniaxial, and its optical axis correlates with its crystallographic axis. Despite the fact that apatite obviously consists of other atoms than tourmaline or quartz, experiments were conducted to check its potential piezoelectric properties. In favor of such experiments were the opinions of researchers [1], suggesting that type 1 collagen in bone trabeculae cannot have piezoelectric properties. Due to all of the above, experimental investigation of apatite piezoelectricity was conducted.

\section{Material and Methods}

Acquiring apatite crystals of adequate purity and size for the planned research turned out to be very complicated. Milky apatite from the Kola peninsula, which are up to several centimeters long, consisted many liquid and gas inclusions.

This property resulted in excluding them from further analysis. Apatite crystals from Morocco were admittedly small in size (Figure 1A), but they were macroscopically clean. They had, however, a series of tiny cracks along the natural cleavage planes, which transpired to be important in further research (Figures 1B, 2A and 2B). Therefore, 
Citation: Pawlikowski M (2017) Biomineralogical Investigation of Apatite Piezoelectricity. Adv Recycling Waste Manag 2: 122. doi: $10.4172 / 2475-7675.1000122$

Page 2 of 6

apatite crystals from Portugal (Figure 3) were finally used for further research. Two plates were cut: one parallel to the optical axis $\mathrm{Z}$ (crystallographic axis $\mathrm{C}$ ), the other perpendicular to optical axis $\mathrm{Z}$ (crystallographic axis C). Both sides of the plates were polished with diamond paste. Two copper electrodes were attached to the opposite sides of the $0.8 \mathrm{~mm}$-thick plates with conductive glue (Figure 4 ).
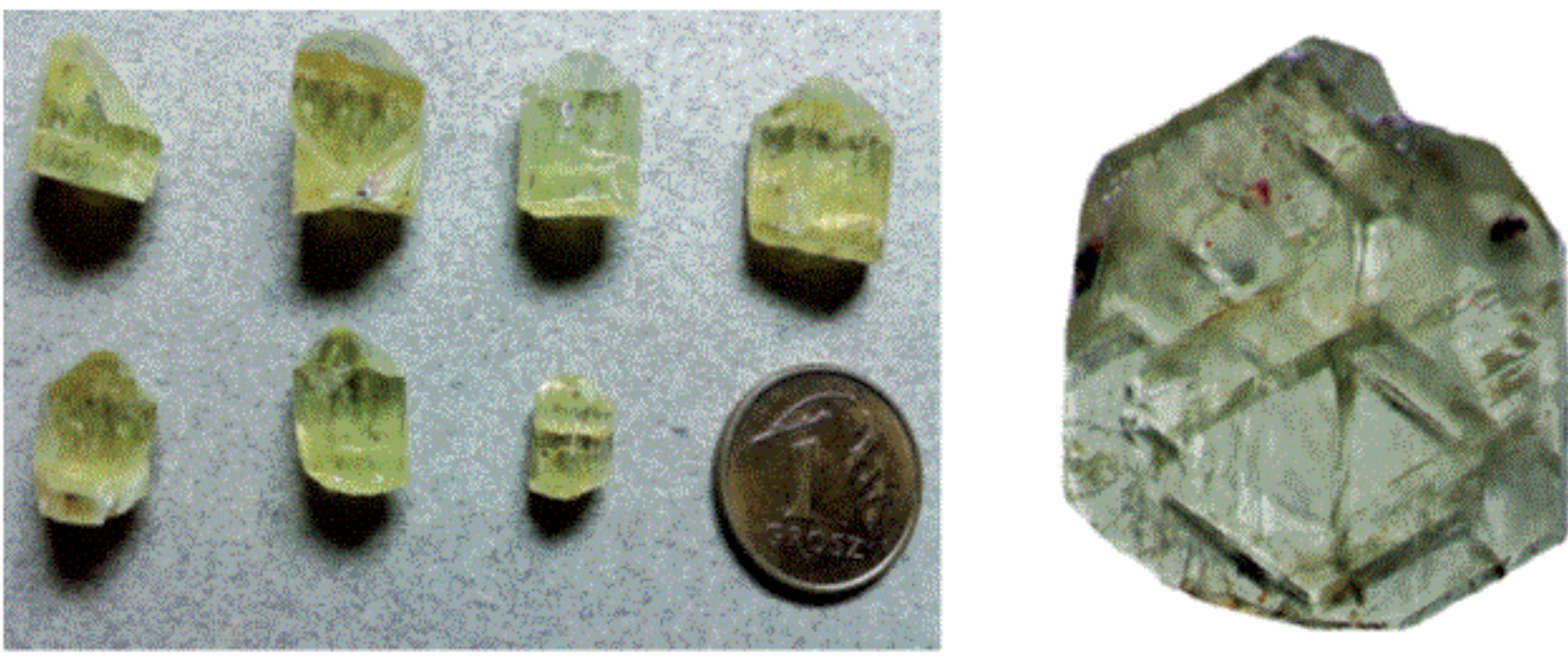

Figure 1: Crystals of apatite from Morocco used in the first experiment (A); picture of a plate of apatite cut perpendicularly to optical axis $Z$ (B). Visible are inclusions and a net of cracks enlarged $\times 10$.
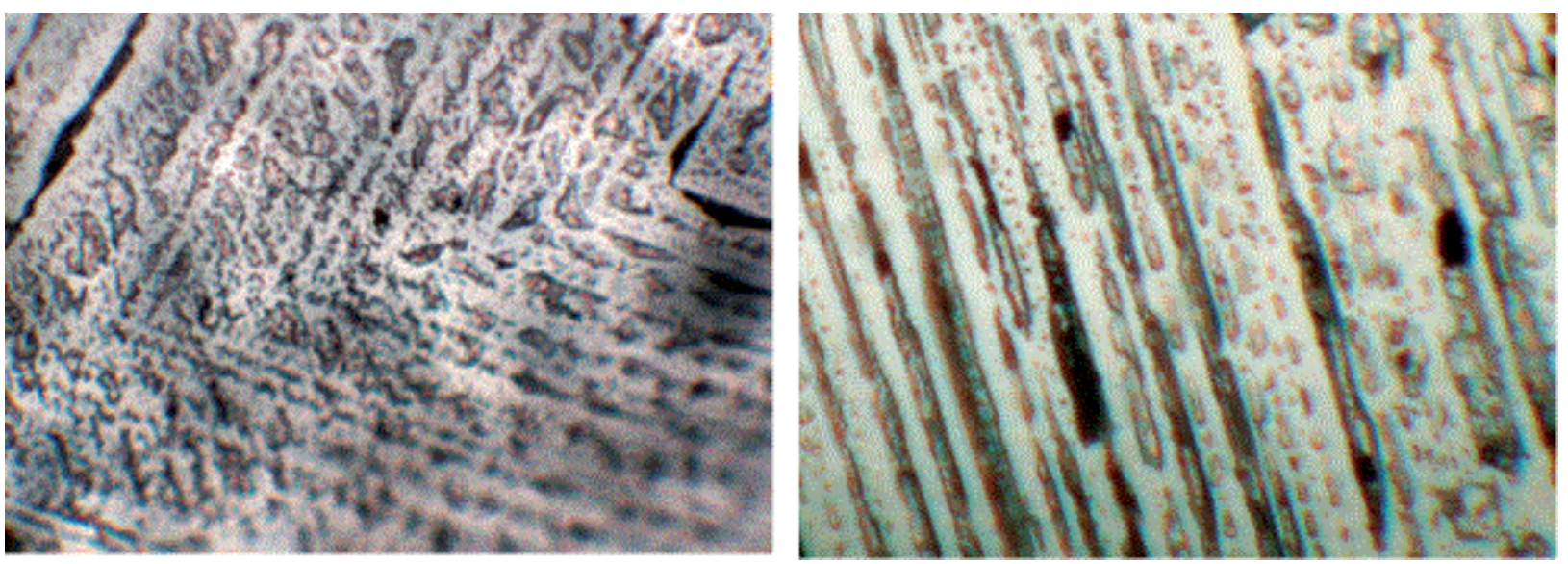

Figure 2: Apatite from Morocco: A- system of inclusions in examined apatite showing stages of crystallization. Polarizing light microscope, 1 polaroid, magnification $\times 200$; B- inclusions orientated parallelly to the walls of the examined apatite crystal. Polarizing light microscope, 1 polaroid, magnification $\times 200$. 
Citation: Pawlikowski M (2017) Biomineralogical Investigation of Apatite Piezoelectricity. Adv Recycling Waste Manag 2: 122. doi:
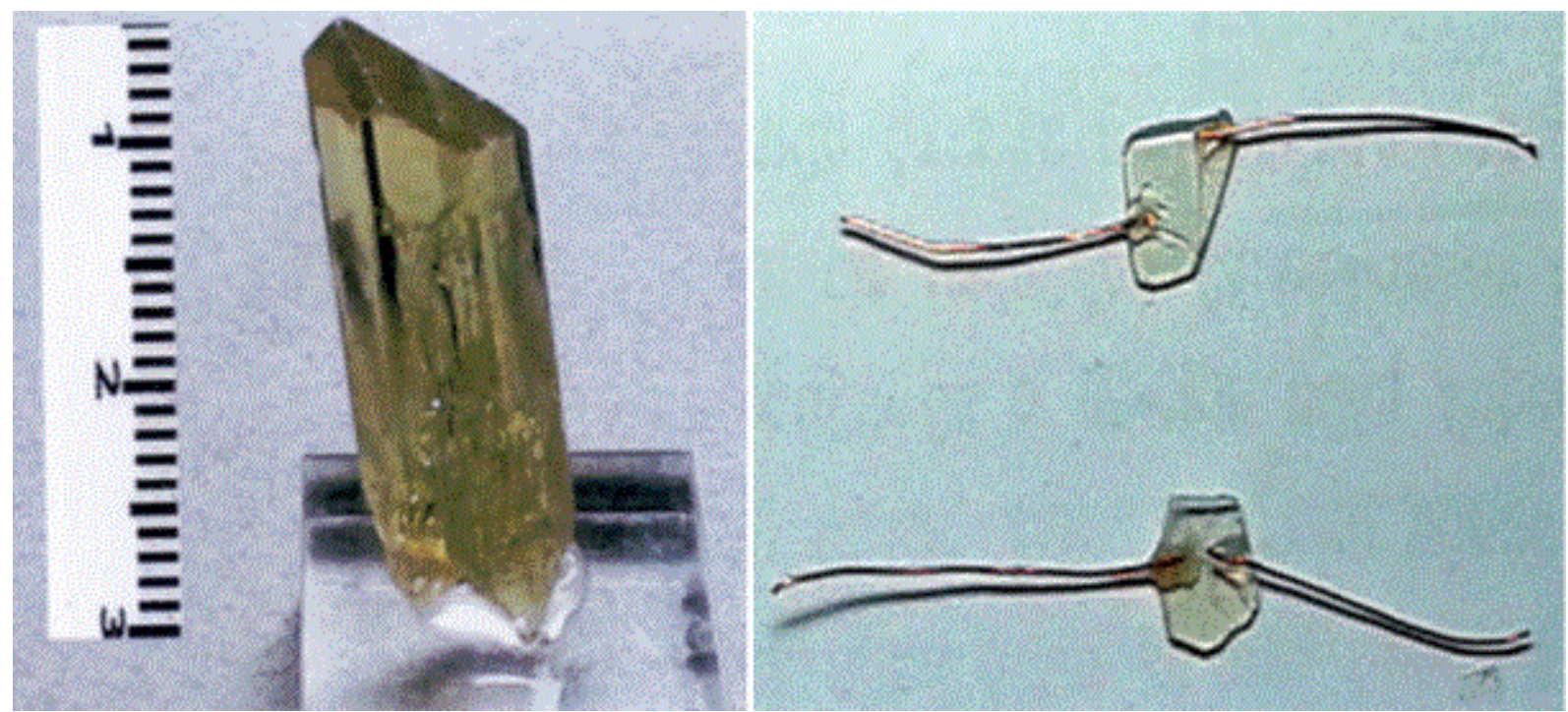

Figure 3: Clean and untracked apatite crystal from Portugal, purchased on the market in Paris, intended for the second experiment regarding piezoelectricity of that mineral (A). plates cut from the clean apatite with attached electrodes, before dusting with gold. Upper plate - cut parallely to optical axis Z (B). Lower plate - cut perpendicularly to optical axis Z. Edges of the plates were coated with paraffin before dusting with gold (Figure 4). After dusting, the paraffin was removed. Thickness of the plates: $0.8 \mathrm{~mm}$. Scale: $\mathrm{cm}$

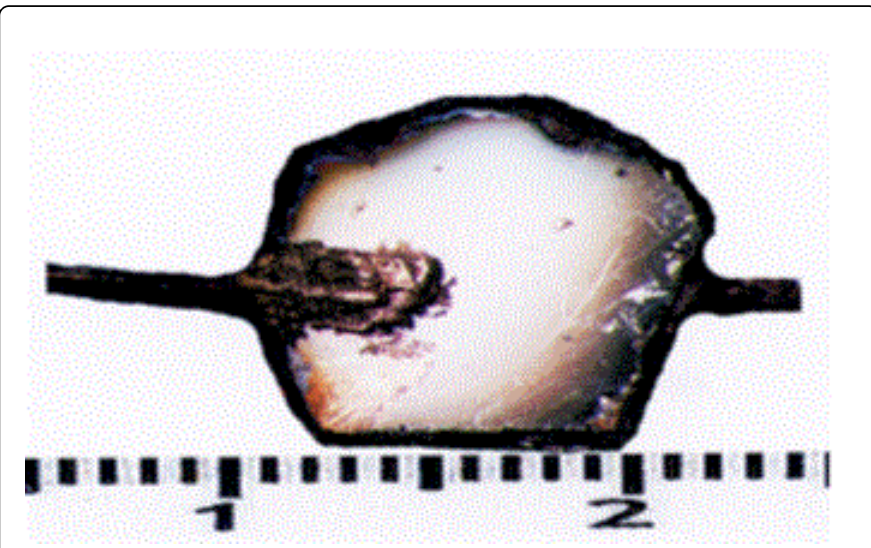

Figure 4: Example image of an appetite plate cut perpendicularly to the optical axis $\mathrm{Z}$ after dusting with gold. The dark edge is a paraffin border; after dusting with gold and removal of the border, upper and lower surfaces of the plates were electrically separated.
After dusting the plates with gold, their electrical measurements were taken. Measurements of the piezoelectricity of the apatite plates were taken with the Emico device (of Polish production) in the EEG room of the Jagiellonian University Hospital in Krakow (Collegium Medicum, Krakow, Lubicz Street- Figure 5A). The plates were attached to the electrodes (Figure 5B). Reactions of the device (changes in currents) on the ends of the plates were recorded, after gently tapping the plates. The measurements were taken at different sensitivity levels (Figure 6). Charts of the currents generated during the experiment are presented below (Figures 6 and 7). Figures 6A and 7A show original charts recorded while gently tapping the crystals. Figures $6 \mathrm{~B}$ and $7 \mathrm{~B}$ show enlarged charts of currents generated while tapping the crystals. Current curves recorded during a hand movement, without tapping, are shown in Figures 6C and 7C. 
Citation: Pawlikowski M (2017) Biomineralogical Investigation of Apatite Piezoelectricity. Adv Recycling Waste Manag 2: 122. doi: $10.4172 / 2475-7675.1000122$

Page 4 of 6
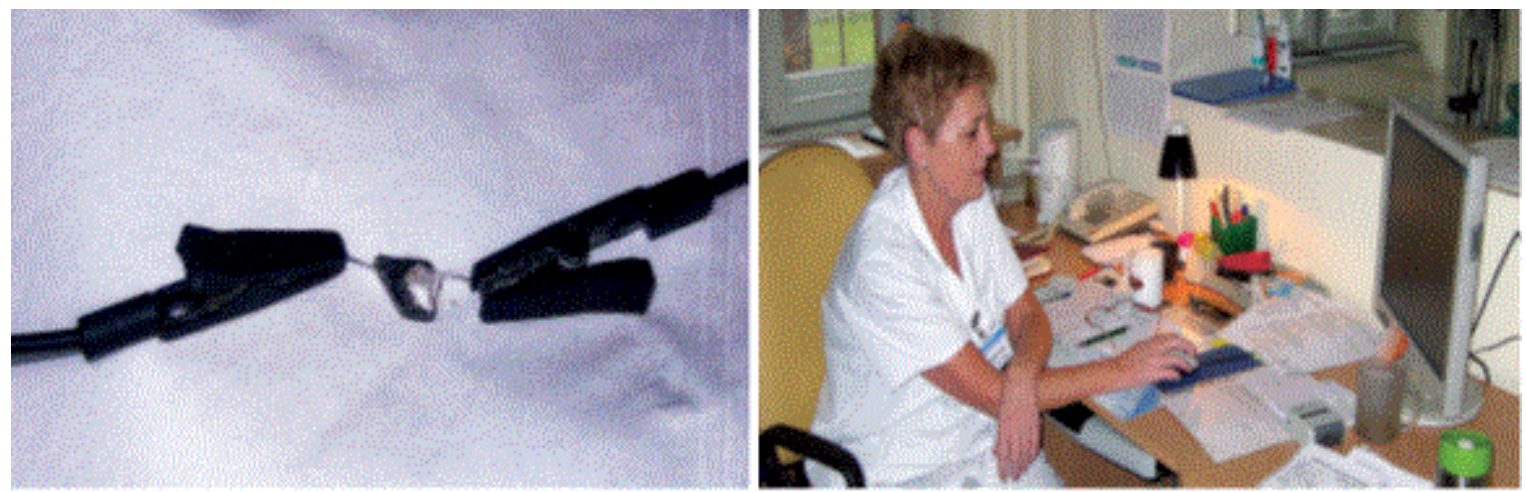

Figure 5: Małgorzata Janik, records the measurements acquired from the EEG device and measurements of the apatite plate cut in parallel to the crystallographic and optical axis Z.
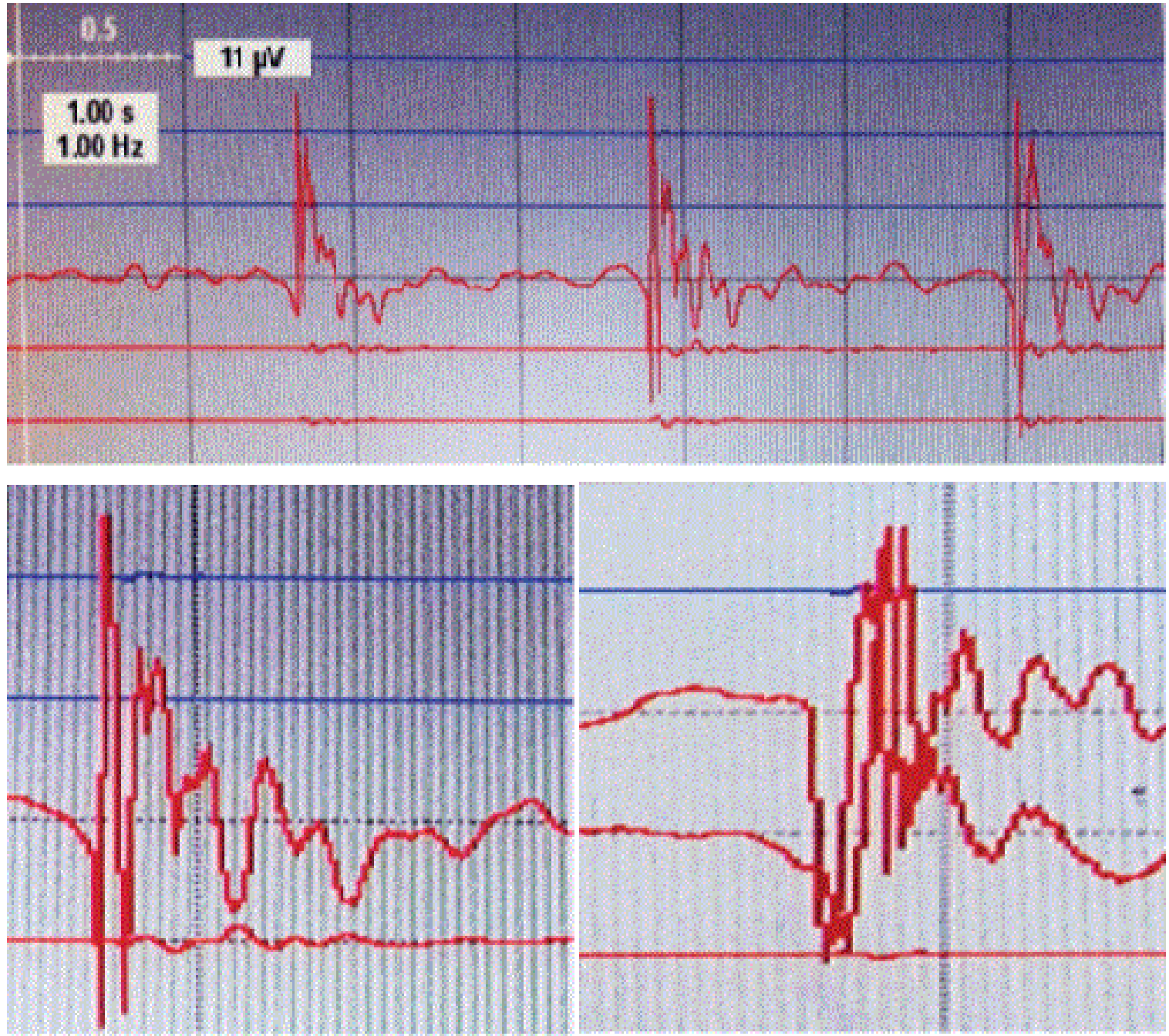

Figure 6: Charts of currents generated by the EEG device while tapping (three times) an appetite plate cut in parallel to the crystallographic axis $\mathrm{Z}$ of the apatite crystal (A). example of the currents chart after tapping the crystal (B). Example of the currents chart recorded during a hand movement $(\mathrm{C})$. 
Citation: Pawlikowski M (2017) Biomineralogical Investigation of Apatite Piezoelectricity. Adv Recycling Waste Manag 2: 122. doi:
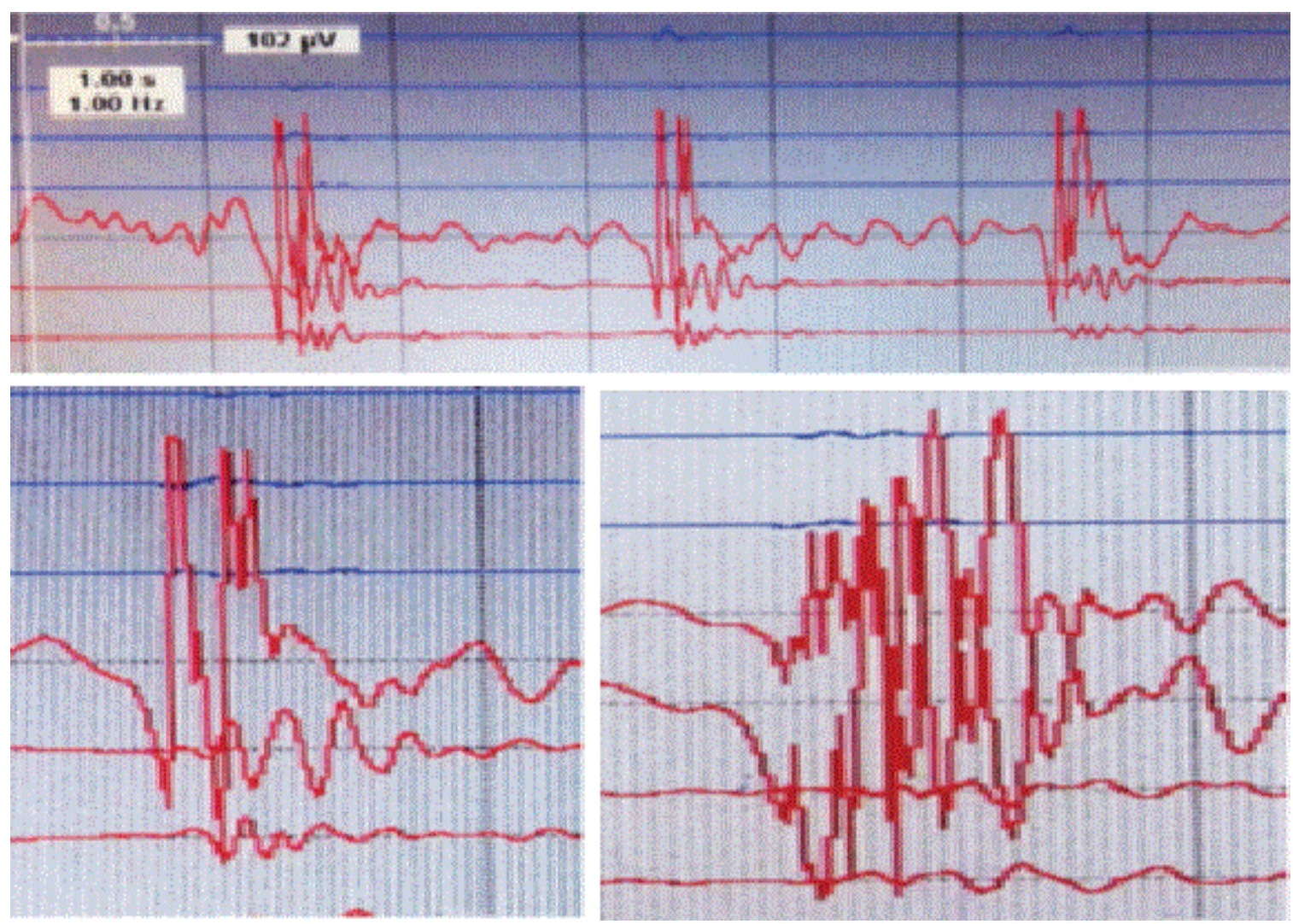

Figure 7: Charts of currents generated by the EEG device during tapping (three times) an apatite plate cut perpendicularly to the crystallographic axis $\mathrm{Z}$ of the apatite crystal (A). example of the currents chart after tapping the crystal (B). example of the currents chart recorded during a hand movement $(\mathrm{C})$.

Detailed observation of the enlarged current scurves for both types of apatite plates shows a slightly different curve between tapping the crystal and only moving the and. Currents curves after tapping the crystal begin with a drop-in voltage, followed by a jump that indicates generating the current, and then a broken but clear, slow decline. Curves registered during a hand movement, without tapping the apatite plate, often (though not always) have a trajectory that is quite similar to the Gaussian one. The phenomenon may be described as a drop-in voltage followed by a systematic rise and slow decline. The conducted experiments indicate that the electricity phenomena recorded by the EEG device are slightly different between tapping the apatite crystals and a hand movement. The character of the currents curves indicates that tapping both types of the apatite plates generates weak current of a voltage between a few and a dozen $\mathrm{V}$.

\section{Conclusion}

Due to lack of funding, both the research and the results should be treated as preliminary and not documented statistically. The goal was to show the phenomena and the investigation methods, and to inspire other researchers to continue the analysis.

The importance of apatite piezoelectricity, due to the significant content of that mineral in bones, is hard to overestimate. Presented results indicate that apatite shows weak piezoelectric properties. Through analogy, we may assume that apatite contained as nanocrystals in the collagen structure of bone trabeculae has such properties as well. Hence the conclusion that the electrical effect observed in bones during their deformation $[6,8,27]$ may be a result of piezoelectric properties of bone apatite. Tensions affecting the hydroxyapatite nanocrystals located in the collagen structure of the bone trabeculae, for instance during movement, are transported through the collagen strands in which the crystals are located. One should assume that despite the nano-size of the apatite crystals rooted in the collagen strands and infinitesimal piezo effects on each crystal, the overall electrical effect in the whole trabeculae, and at the same time in the whole bone, is a sum of separate effects occurring on separate crystals. Piezo effect of a single nanocrystal of bone hydroxyapatite is not measurable using present day methods, whereas the adding up of the effect was investigated and measured mainly in the long bones $[6,8,17,28]$.

There were also attempts to use electrical phenomena to accelerate fracture healing $[23,24]$. In that case, the phenomena were not analyzed in relation to piezoelectricity, which, one may suppose, played an important role in stimulating fracture healing through vibration of the hydroxyapatite nanocrystals. It is also possible that a series of factors, both external and internal (for example, increase in atmospheric and arterial pressure), may influence mechanical deformations of bone and its electrical potentials [31]. External electric field also influences mechanical behavior of the piezoelectric nanocrystals of apatite. Piezoelectric qualities of crystals, including the 
apatite we analyzed, allow for transformation of electrical energy into mechanical, and mechanical into electrical. Mechanical impact on apatite crystals, through various tensions connected with the bone structure, may cause the creation of electrical currents in the bone apatite crystals, i.e., in bones. Simultaneous electrical impact, e.g., Changes in electromagnetic field, may probably lead to mechanical deformations of the bone apatite crystals, and as a result, the whole bones. That entitles us to claim that thanks to its discovered piezoelectric qualities, apatite, including bone apatite, plays a role of a transformer. Transformation of mechanical energy to electrical and electrical to mechanical by the bone apatite is probably a stimulation factor for many phenomena, not just in bones, but in the whole body [30]. Those phenomena should be an object of further multidirectional research.

\section{References}

1. Ahn AC, Grodzinsky AJ (2009) Relevance of collagen piezoelectricity to Wolffs law, a critical review. Med Eng Phys 31: 733-741.

2. Anderson JC, Eriksson C (1968) Electrical properties of wet collagen. Nature 21: 166-168.

3. Aschero G, Gizdulich P, Mango F, Romano SM (1996) Converse piezoelectric effect detected in fresh cow femur bone. J Biomech 29: 1169-1174.

4. Basset CA, Becker OR (1962) Generation of electric potentials by bone in response to mechanical stress. Science 137: 1063-1064.

5. Cerquiglini S, Cignitti M, Salleo A (1967) On the origin of electrical effects produced by stress in the hard tissues of living organisms. Life Science 6: 2651-2660.

6. Fukada E, Yasuda I (1957) On the Piezoelectric Effect of Bone. J Phys Soc Jpn 12: 1158-1162.

7. Williams WS, Breger L (1974) Analysis of stress distribution and piezoelectric response in cantilever bending of bone and tendon. Ann N Y Acad Sci 238: 121-130.

8. Szewczenko J (2005) Electrical phenomena in the long bones. Overview Electrotechn 81: 94-97.

9. Curie J, Curie $\mathrm{P}(1880)$ Development, by pressure, of polar electricity in hemihedron crystals with inclined faces Submitted by Mr. Friedel. Proceedings of the Acad of Sci 91: 294-295.

10. Curie J, Curie P (1880) On polar electricity in hemihedral crystals with inclined faces. Submitted by Mr. Desains. Proceedings of the Academy of Sciences 91: 383-386.

11. Curie J, Curie P (1881) Laws of release of electricity by pressure, in tourmaline. Presented by M. Friedel. Reports of the Acad of Sci 92: 186-188.

12. Curie J, Curie P (1881) The hemihedron crystals with inclined faces, as constant sources of electricity. Submitted by M. Desains. Proceedings of the Academy of Sciences 93: 204-207.
13. Curie J, Curie P (1881) On the electric phenomena of tourmaline and hemihedral crystals with inclined faces. Submitted by Mr. Friedel. Proceedings of the Academy of Sciences 92: 350-353.

14. Curie J, Curie P (1881) Contractions and dilatations produced by electric voltages in hemihedral crystals with inclined faces, Submitted by M. Friedel. Proceedings of the Academy of Sciences 93: 1137-1140.

15. Curie J, Curie P (1882) Electrical deformation of quartz. Submitted by Mr. Desains. Proceedings of the Academy of Sciences 95: 914-917.

16. Curie J, Curie P (1882) Electrical phenomena of hemihedron crystals with inclined faces. J of Physics 2nd Series, 1: 245-251.

17. Korostoff E (1979) A linear piezoelectric model for characterizing stress generated potentials in bone. J Biomech 12: 335-347.

18. Curie J, Curie P (1893) Piezoelectric Quartz. Phil Mag 36: 340-342.

19. Curie J, Curie P (1889). Electrical expansion of quartz. J of Philosophy (2nd series) 8: 149-170.

20. Anderson JC, Eriksson C (1968) Electrical properties of wet collagen. Nature 21: 166-168.

21. Johnson MW, Chakkalakal DA, Harper RA, Katz JL (1980) Comparison of the electromechanical effects in wet and dry bone. J Biomech 13: 437-442.

22. Żuk T, Dziak A, Gusta A (1980) Basics of orthopedics traumatology, Warsaw, PZWL pp- 42.

23. Jacobson-Kram D, Tepper J, Kuo P (1997) Evaluation of potential genotoxicity of pulsed electric end electromagnetic fields used for bone growth stimulation. Mutation Research 388: 45-57.

24. Starkebaum W, Pollack SR, Korostoff EJ (1979) Microelectrode studies of stress-generated potentials in four-point bending of bone, J Biomed Mater Res 13: 729-51.

25. Weinbaum S, Cowin SC, Zeng Yu (1994). A model for the excitation of osteocytes by mechanical loading-induced bone fluid shear stresses, J Biomech 27: 339-360.

26. Weigert M, Wehahn C (1977) The influence of electric potentials on plated bones. Clin Orthop 124: 20-30.

27. Yasuda I (1953) Fundamental aspects of fracture treatment. J Kyoto Med Soc 4: 395-406.

28. Basset CA, Becker OR (1962) Generation of electric potentials by bone in response to mechanical stress. Science 137: 1063-1064.

29. Salzstein RA, Pollack SR, Mak AFT, Petrov N (1987) Electromechanical potentials in cortical bone. J Biomech 20: 261-270.

30. Pawlikowski M, Niedźwiedzki T, Mineralogia kości (2002) Mineralogy of bones. Polish Acad Sci Kraków. pp -111.

31. Garland DE, Moses B, Salyer W (1991) Long-term follow-up of fracture nonunions treated with PEMFs. Contemp Otrhop 3: 295-302. 